\title{
Aprendizaje informal y prácticas agroecológicas en el contexto de una huerta
}

\author{
Informal learning and agroecological practices in \\ the context of an orchard
}

\author{
Candela Perroni Gasull ${ }^{1}$; Emilia Sago Herrador ${ }^{2}$; Rocío Belén Martín ${ }^{3}$
}

\begin{abstract}
Resumen
El siguiente trabajo es abordado desde un enfoque etnográfico, y tiene como propósito conocer aspectos relevantes del aprendizaje co-construido a través de prácticas agrícolas en el contexto informal de una huerta, ubicada dentro del territorio de una universidad nacional de Argentina, configurada de forma autónoma y horizontal. A través del análisis de las narrativas de los huerteros que participaban con regularidad en la huerta, se elaboraron categorías de análisis sobre los rasgos predominantes del aprendizaje agroecológico, a saber: (1) trayectorias de los participantes, (2) aprendizaje como práctica comunitaria, (3) aspectos personales del aprendizaje, (4) prácticas generadoras de aprendizaje y (5) dimensión política-social.
\end{abstract}

Palabras Clave: Agroecología; aprendizaje; trayectorias, huerta.

\begin{abstract}
The following work is approached from an ethnographic approach, and its purpose is to know relevant aspects of co-constructed learning through agricultural practices in the informal context of an orchard, located within the territory of a national university in Argentina, configured autonomously and horizontal. Through the analysis of the narratives of the orchards that regularly participate in the orchard, categories of analysis were elaborated on the predominant traits of agroecological learning, a saber: (1) trajectories of the participants, (2) learning as community practice, (3) personal aspects of learning, (4) learning-generating practices and (5) political-social dimension.
\end{abstract}

Keywords: Agroecology; learning; trajectories; orchard.

\footnotetext{
${ }^{1}$ Facultad de Ciencias Exactas, Físicas y Naturales, Universidad Nacional de Córdoba. E-mail: cande.perroni@gmail.com

${ }^{2}$ Facultad de Ciencias Exactas, Físicas y Naturales, Universidad Nacional de Córdoba. E-mail: emisagoh@gmail.com

${ }_{3}^{3}$ Facultad de Ciencias Exactas, Físicas y Naturales, Universidad Nacional de Córdoba. E-mail: rbmartin@unc.edu.ar

Submetido em: 26/06/2020 - Aceito em: 29/06/2020
} 


\section{Introducción}

"Hubo un sol que supo arder sin consumirse en su deber.

Ser que daba tiempo al deseo.

Visitante que asomado entre placeres acompaña $y$ sin querer descubre, mientras enseña queriendo.

Conocedor que alimenta sin recetas.

Textos escritos en ojos que cuentan.

Manos que acompañan traduciendo el fuego, refugiadas en el seno del más fértil suelo."

Candela Perroni Gasull

En los placeres y en la receta de investigar, actualmente los contextos y aprendizajes informales están echando raíces, esto lleva a investigar acerca de los aspectos relevantes del aprendizaje construidos de manera colectiva a través de prácticas agroecológicas colaborativas y cooperativas en un contexto informal.

El estudio se llevó a cabo en el marco del Profesorado en Ciencias Biológicas, de la Facultad de Ciencias Exactas, Físicas y Naturales de la Universidad Nacional de Córdoba, buscando investigar e incorporar innovaciones pedagógicas que contemplen otros contextos, como los informales, para re-pensar la formación de los profesionales de la educación, quienes ya no solo deben ajustar sus interacciones a ámbitos formales, sino que, junto con la complejización y la extensión de contextos deben ser conocedores y conectores entre diversos ambientes y formas de aprendizaje (MARTÍN, PALOMBO, PERRONI GASULL, PAREDES MALDONADO, SAGO HERRADOR Y CHIAPERO, 2019).

La pregunta de DUSSI, FLORES \& BARRIONUEVO (2014): “¿Es el pensamiento multidimensional un pre-requisito para entender la agroecología o es el resultado de nuestras experiencias agroecológicas lo que nos permite comprender el todo?" sugiere indagar sobre la potencialidad de extrapolar capacidades aprehendidas en un contexto informal, como el de la huerta, y cómo estas capacidades podrían aportar a filosofías que promuevan la vida en comunidad de manera más armónica, enseñando a los individuos a respetar pacientemente los tiempos cíclicos de la naturaleza, mediante el contacto y vínculo con la fuente y origen de los alimentos, en estrecha relación con una visión holística de su huella en el quehacer diario. En concordancia, existe un informe realizado para la UNESCO de la Comisión Internacional para la Educación del siglo XXI presidida por DELORS en 1996 , denominado "La educación encierra un tesoro", donde se explicita que el aprendizaje durante toda la vida, que se asocia al proceso de aprendizaje en contextos informales -debido a que su motor existencial es alimentado por intereses motivacionales propios-, es como el "latido" de una sociedad que se basa en cuatro pilares: Aprender a conocer, aprender a hacer, aprender a vivir juntos y aprender a ser. El informe extiende su ideal de aprendizaje a una escala social en la que todas y cada una de las personas puedan aprender de acuerdo a sus necesidades e intereses individuales, en 
cualquier lugar y en cualquier momento de una manera libre, flexible y constructiva. Este ideal de aprendizaje propuesto por DELORS (1996) se abraza con prácticas sustentables en comunidades reducidas donde el intercambio y la participación son el eje central del desarrollo de múltiples tareas para alcanzar objetivos comunes entre varias personas; esta apreciación da lugar a pensar que quizás sea ese el marco que posibilita el aprendizaje en la huerta. A partir de ello surgen algunos interrogantes: ¿Podría ser la educación basada en este tipo de aprendizajes la principal fuerza capaz de promover la transformación del mundo?. ¿No deberían los aprendizajes cotidianos y situados ser resignificados? ¿No aportan estos saberes un modo de habitar el mundo de una manera más armónica, empática y sustentable?

En este sentido, se plantea la idea de que el desarrollo intelectual no se encuentra separado del emocional, ya que estos aspectos responden a un contexto histórico y sociocultural en el que confluye la acción y la interacción social (MEJÍAS ARAUZ, 2005) y a la vez, es en contextos informales donde se co-construyen o reproducen las estructuras socioeconómicas y culturales del que aprende.

\section{Marco Teórico}

En contextos informales el aprendizaje acontece indiferenciado y subordinadamente a otros procesos sociales; es decir, cuando está inmerso en otras realidades culturales (TRILLA, GROS, LÓPEZ Y MARTÍN, 2003), no relacionado a los formatos que presupone la educación formal. Este rasgo se enraiza de forma consistente y profunda en valores, actividades y procesos pertinentes a esa otra realidad cultural en la que está inmerso el o la que aprende, generando a través de sus prácticas un vínculo con su dimensión política en un contexto determinado. La educación informal puede asociarse al concepto de aprendizaje para la vida o bien aprendizaje permanente, ya que las personas adquieren y construyen conocimientos, habilidades y actitudes mediante las experiencias cotidianas y su relación con el medio ambiente (MARTÍN, 2014).

El aprendizaje informal es poco entendido, poco atendido y con frecuencia tratado con ambigüedad, ya que existe confusión acerca de este concepto relacionado comúnmente con otros como el de aprendizaje no formal, ignorando en esa ambigüedad que estos tipos de aprendizaje no comparten expectativas ni supuestos culturales asociados. En primer lugar, el aprendizaje informal carece de programación de acciones educativas (MARTíN, 2019) y se caracteriza fundamentalmente por lo desintencionado y espontáneo de su acontecer, ya que no pretende cumplir con un objetivo educativo preciso. Otro rasgo característico de esta forma de aprender es su carácter de universal en lo que refiere a la capacidad -evolutivamente adquiridade aprender inherente a los seres humanos y a otros seres. Así, la definición más amplia de aprendizaje informal, comprende la falta de organización en lo que refiere a la intención prescriptiva de enseñanza, donde el proceso de aprendizaje es espontáneo y/o autodirigido y ocurre por medio de la experiencia, al seguir una ruta elegida y controlada por la persona que aprende, por voluntad, motivación e interés propio. El estudio de esta forma particular de aprendizaje, posibilita la profundización en el conocimiento de procesos de aprendizaje y

REnBio - Revista de Ensino de Biologia da SBEnBio - ISSN: 1982-1867 - vol. 13, n. 1, p. 206-222, 2020 
desarrollo cognoscitivo, así como también de algunos procesos de socialización de saberes. (MEJÍA ARAUZ, 2005).

Un concepto y eje transversal en las prácticas huerteras es el de soberanía alimentaria. Según el Movimiento Campesino Internacional, Vía campesina, la soberanía alimentaria es el derecho de los pueblos a definir su política agraria y alimentaria, otorgándole dentro de esta definición un lugar prioritario a las producciones locales de alimento para la población, así como también el derecho de los propios agricultores de producir alimentos y el de los consumidores a decidir lo que quieren consumir, conociendo cómo y quién lo produce entre otras cuestiones de diferentes magnitudes involucradas en este concepto (VÍA CAMPESINA, 2020). En esta dirección, MEJÍA ARAUZ (2005), añade algo interesante al respecto, en la relación al aprendizaje con valores y creencias preexistentes, afirmando que las raíces de estos valores se encuentran situadas en un marco sociocultural, siendo esta una de las razones por las que el aprendizaje informal resulta estimulante para quienes voluntariamente se disponen a construirlo individual o socialmente.

ROGOFF (2003) propone un modelo donde el aprendizaje es una transformación de la participación de la persona en una actividad de práctica o de aprendizaje compartida por una comunidad; en esta concepción, se considera que la evidencia de aprendizaje no es un producto en el sentido de la posesión de conocimiento o de habilidades por el individuo, sino la transformación gradual de la participación la que muestra que la persona se va involucrando cada vez más y asumiendo más responsabilidad en las actividades en que colabora con otros, a la vez que pone de manifiesto de esta forma, mayor dominio y conocimiento de la actividad en cuestión. Cuando los participantes logran involucrarse en la acción, se da una inmersión en una estructura y organización social y cultural donde la actividad que se aprende es relevante para la comunidad, en la que hay participantes con mayor grado de conocimiento y experiencia que guían y fomentan la participación de los aprendices.

\section{Metodología}

La investigación de tipo exploratoria se enmarca en un enfoque etnográfico, que tiene como objetivo principal conocer aspectos relevantes del aprendizaje construido a través de prácticas en el contexto informal de una huerta. Este enfoque -etnográfico- constituye una concepción y práctica de conocimiento que busca comprender los fenómenos sociales desde la perspectiva de sus miembros (entendidos como "actores", "agentes" o "sujetos sociales"). Respondiendo a la descripción; que a su vez responde a tres niveles de comprensión: primario o "el reporte", informa que ha ocurrido (el qué); "la explicación" o comprensión secundaria que alude a sus causas (el porqué); y "la descripción" o comprensión terciaria que responde a que sucedió desde la perspectiva de sus agentes (el cómo es para ellos). En este sentido, los agentes informantes son claves, solo ellos pueden dar cuenta de lo que piensan, sienten, dicen y hacen con respecto al accionar que los convoca. (GUBER, 2011) 
Siguiendo con la descripción sobre la etnografía de GUBER (2011), dar cuenta de lo que sucede en cada territorio sirve para dar cuenta lo más genuinamente posible de una práctica o noción.

Durante el año 2019, se analizó la narrativa de los participantes de la huerta a través de una entrevista semiestructurada y en profundidad, diseñada con anticipación. Este número de cuatro participantes brindó la posibilidad de realizar entrevistas distendidas con cierta amplitud en la confección de preguntas, apelando a respuestas abiertas para que los entrevistados puedan explayarse al comunicar su experiencia de aprendizaje.

Los entrevistados fueron participantes de una huerta que se construyó aproximadamente en 2016, de manera escalonada, con pequeños acercamientos y contribuciones de diversos actores que desempeñan funciones disímiles dentro de la institución $\mathrm{IMBIV}^{4}$ y por fuera de ella (ej. Estudiantes de Biología). En un primer momento, fue la creación de un compost la actividad que condujo a los primeros actores a concurrir al espacio con cierta periodicidad. Posteriormente, la mixtura entre la tierra de relleno y la tierra fértil producida por la compostera, dio a luz los primeros canteros.

La suma de los relatos permite reconocer de manera abarcativa las prácticas comunitarias y dinámica de trabajo colaborativo entre distintos actores, así como también cuales son los aquellos elementos que se entrelazan en los procesos de aprendizaje social y situado de la huerta. Contar con las experiencias y relatos de cada uno posibilita abordar el análisis de manera integral. Los contextos informales dan lugar a que los participantes posean tanto intereses, como prácticas de acción de diferentes, lo que da cuenta del valor intrínseco de cada experiencia y relato para este estudio.

Además de las entrevistas, se hizo tanto necesario como inherente al estudio realizar notas de campo de tipo etnográficas. Siendo esta una herramienta que brinda apoyo a la memoria sobre lo que fue observado y percibido durante la inmersión en el territorio, recreando a través de estas notas escenarios y componentes relevantes de la acción que fueron utilizados en el posterior análisis.

\section{Análisis Y Resultados}

El análisis de los relatos se realizó en función de cinco categorías que se describirán a continuación.

\subsection{Trayectorias de los participantes}

En primer lugar, se indagó sobre cómo fue la llegada al espacio y sobre la participación en otras huertas o espacios similares. Mediante estos relatos, se intentó dilucidar cuál fue la motivación que los llevó a la búsqueda de contextos informales para aprender sobre el trabajo

\footnotetext{
${ }^{4}$ Instituto Multidisciplinario de Biología Vegetal del Conicet y la Universidad Nacional de Córdoba, Argentina. REnBio - Revista de Ensino de Biologia da SBEnBio - ISSN: 1982-1867 - vol. 13, n. 1, p. 206-222, 2020
} 
en la tierra. También se buscaron posibles relaciones entre experiencias previas en huertas, prácticas actuales y sobre lo compartido y adquirido en vinculaciones por fuera del espacio, esto es, en otros entornos en los que se vean involucradas prácticas similares a las que se llevan a cabo en la huerta, atendiendo también al componente afectivo en torno a las posibles relaciones encontradas; y por último, las visiones o expectativas a futuro.

En relación a esta categoría, en la mayoría de los relatos los entrevistados expresaron que su actividad en la huerta fue la iniciación de su participación en espacios de trabajo colectivo y cooperativo. A su vez, hacen mención de algunas prácticas relacionadas con acercamientos a pequeñas huertas en sus casas o al cuidado de algunas plantas dadoras de alimentos por algun encuentro azaroso con las mismas:

Tenía desde hace unos años en casa unos plantines que habían crecido... Un zapallo, un tomate por ahí pero no era una huerta instalada. Como proyecto colectivo fue la primera vez. (Entrevista huertero $N^{\circ} 1$ )

Además, de todos los relatos se desprende como factor común para haber concretado el acercamiento al espacio, la mediación de algún vínculo afectivo con personas que propiciaron el encuentro con al espacio en general y las prácticas en particular. En este sentido, algunos entrevistados hacen mención de su llegada a través de redes de amistad y de manera colaborativa hacia quien abre las puertas. También se pudo identificar como un aspecto motivacional de participación, un interés en torno a las prácticas de huerta que solo requería encontrar un suelo en la ciudad para enraizar:

A mi es algo que me acompaña hace bastantes años, con mi abuelo fue con la persona con la que conocí una huerta por primera vez (...) cuando me vine a Córdoba todo eso se vino conmigo, todas esas ganas. Primero fue súper difícil porque vivía en un departamento re chiquito y después me fui acomodando en la ciudad y buscando los espacios para hacer huerta (Entrevista huertero $N^{\circ} 2$ )

Todos los entrevistados reconocen que el haber construido o participado de la huerta del instituto, fue el disparador para continuar gestando y contribuyendo en espacios similares. Actualmente todas las personas entrevistadas se encuentran participando en otros espacios en los que se llevan a cabo actividades vinculadas a la agroecología, el trabajo digno y economías alternativas, así como también resaltan la importancia de articular con otras huertas comunitarias y con todas aquellas personas que se interesen por este tipo de prácticas:

Un poco también nuestra impronta y esperanza es que esos vínculos y articulaciones sean cada vez más fuertes para que todos los espacios de agricultura urbana se sigan sosteniendo y vayan creciendo en valor (Entrevista huertero $N^{\circ} 2$ ) 


\subsection{Aprendizaje como práctica comunitaria}

Esta dimensión considera todos aquellos aspectos del aprendizaje que se ponen en juego mediante el vínculo con otro; por ejemplo analizar el grado de implicación de cada participante para con los quehaceres del espacio y qué percepción tienen sobre la dinámica horizontal de las prácticas llevadas a cabo, si fluctúa esta horizontalidad frente a alguien que demuestre saber más o cómo se construye frente a esta posibilid. También se consideró la posible existencia de planificación previa o la espontaneidad de las prácticas, y sobre la posibilidad de tareas asignadas, entre otras.

En todos los casos, los huerteros pusieron gran énfasis en el vínculo con otros al momento de hablar de su aprendizaje, considerando los aspectos comunitarios de la labor y la comunicación con otras personas y espacios similares como parte fundamental del proceso. En palabras de los entrevistados:

No está muy claro quien enseña y quien aprende, sino que ese vínculo es algo mucho más fluido entre las personas (...) compartir el trabajo en la tierra es poder ser consciente de que todas las personas pueden ser maestras de algo en algún momento y tienen algo para compartir y enseñar, y todos también tenemos algo siempre para aprender. (Entrevista huertero $N^{\circ} 2$ )

Toda la gente que paso por aca dejo datos (Entrevista huertero $N^{\circ} 1$ )

En la feria agroecológica también he aprendido con los productores (...) ver plantas nuevas, conocer semillas nuevas (Entrevista huertero $N^{\circ} 4$ )

La huerta tiene a simple vista, como si estuviera hecha con basura, no sé. Con un montón de material de descarte. Bueno y eso es algo que en los otros espacios fuimos viendo que se replicaba o diciéndoles también: 'che, nosotros resolvimos esto de esta forma', por que bueno por ahi hay cosas que requieren cierta infraestructura o mas bien es como la necesidad humana de transformar, transformar, transformar porque también vino gente acá que nos habló de técnicas más relajadas donde uno no ingresa tanta energía... (Entrevista huertero $\left.N^{\circ} 1\right)$

En un sentido aún más holístico del aprendizaje se percibe, a través del análisis de las narrativas, el rol central de la relación entre los participantes con la tierra y con la complejidad del ecosistema en su totalidad, ya que todos asumen éste vínculo como uno más dentro de la matriz horizontal y comunitaria del aprendizaje, manifestando que es la propia tierra de quién y con quien se aprende en primera instancia.

La tierra te va diciendo que tenes que hacer (Entrevista huertero $N^{\circ} 3$ )

(se aprende...) primero de la tierra sin dudas (...) Y después los pares con los que estás trabajando. (Entrevista huertero $\left.N^{\circ} 4\right)$ 
En este sentido podemos decir que es la tierra la principal guía en la estructuración de los aprendizajes colectivos que tienen lugar la huerta.

Volviendo al eje central de esta investigación, contextos informales de aprendizaje, resulta tanto relevante como de gran interés resaltar la superposición intrínseca (por superposición territorial y la trayectoria particular de los entrevistados) que existe en el escenario de esta investigación. Siendo la huerta un espacio en el que se desarrollan prácticas espontáneas y cooperativas entre pares pero en consonancia con la existencia de saberes y formas propias de los sistemas académicos de educación, se puede denotar como, en algunos casos, estos saberes se entremezclan en las narrativas. A continuación, algunos fragmentos que dan cuenta de lo anterior:

Si bien la gente que participa de la huerta al estar relacionada con la carrera de biología, más allá de no estar relacionados con las plantas en sí mismas, tiene un conocimiento de las plantas, por ahi todos tienen alguna informacion con respecto a cómo cultivar un vegetal y el intercambio es más horizontal (...) Después fue cuestión de cada uno de ir aprendiendo más en otros espacios sobre cómo cultivar, como tener una huerta y luego en el espacio que encontrás, que es en la huerta, compartir eso de distintos lugares pero sí bastante horizontal y participativo. (Entrevista huertero $N^{\circ} 3$ )

(Aprendo...) de la complejidad de todo, solo jugamos a querer controlar cosas pero todas las variables que se te escapan. Aparte tengo mucho el chip del biólogo que hace experimentos constantemente y te pones a probar cosas distintas pero imposible, ni siquiera es la prueba lo que está ocurriendo, está pasando todo (...). (Entrevista huertero $N^{\circ} 1$ )

Los fragmentos posteriores sustentan nuestra intención de otorgarle al aprendizaje en contextos informales la relevancia que amerita, en tanto generador de aprendizajes para toda la vida al hacer referencia a la conciencia, a los derechos, al vivir en comunidad, a la responsabilidad social y a muchos otros aprendizajes que de esto se desprenden, los cuales solo son posibles de construir de manera colaborativa:

Hay muchas cosas sobre las cuales no tenemos información previa... Cómo respetar al otro, de cómo no jerarquizar, de cómo trabajar codo a codo, horizontal, ese es el trabajo en el que más agua hacemos pero en el que más rosqueamos, discusiones, distancias, por esta cuestión de no tener un jefe a quien decirle 'che solucionanos el problema', naturalmente se dio tratar de trabajar así, ese es un hiperaprendizaje (Entrevista huertero $N^{\circ} 1$ )

Volviendo a una de las preguntas iniciales que guían esta investigación: “¿Es el pensamiento multidimensional un pre-requisito para entender la agroecología o es el resultado de nuestras experiencias agroecológicas lo que nos permite comprender el todo?", uno de los entrevistados comentó lo siguiente en relación a su participación en otro espacio de mayor magnitud del cual hoy forma parte: 
Estamos aprendiendo a producir respetándonos, a producir con trabajo digno, siendo solidarios, cooperativos con nosotres, eso incorpora la agroecología, que no es solamente producir el alimento sino entender que hay otra forma de relacionarse entre el trabajo y los consumidores. (Entrevista huertero $\mathrm{N}^{\circ} 2$ )

Este pedazo de tierra es de cada persona que vino acá y le puso energía (Entrevista huertero $\left.N^{\circ} 1\right)$

Estos relatos dan certeza a las inquietudes iniciales de esta investigación en torno a la potencialidad de los saberes construidos colaborativamente como fuente para la transformación social y la construcción de modos de habitar el mundo de una manera más sustentable y equitativa.

\subsection{Aspectos personales del aprendizaje:}

En esta categoría se enfatiza en los procesos personales del aprendizaje en este contexto. Se busca reconocer aspectos motivacionales de los participantes, sobre sus expectativas, procesos metacognitivos, reflexiones sobre sus prácticas cotidianas en el espacio y sobre la influencia de las mismas asociadas a sus aprendizajes que resultan extrapolables a la vida cotidiana.

Los aspectos motivacionales tienen mucha relación con los atributos colaborativos del aprendizaje de estas práctica. Hemos visto reflejado en las palabras de los entrevistados, que el entusiasmo principal surge de trabajar con otros. Incluso la labor personal de cada uno de ellos, fue tomando forma y constancia frente a la intención de proyectar tareas a largo plazo, acompañados y atravesados por un proyecto común.

Venir a trabajar acompañado es re diferente, lo que lo hacía con mucho más entusiasmo y mucha más frecuencia y bueno, despues tambien fuimos cambiando de ambientes (...) a principio de año estábamos organizades: 'voy los lunes, vos vas los miércoles'... (Entrevista huertero $N^{\circ} 1$ )

También cabe destacar que los momentos de desmotivación para algunos de ellos se vinculan con momentos de desorganización de lo colectivo:

"Uno se comienza a alejar cuando encuentra el espacio desorganizado, si uno ve que vuelve y no está el mismo esfuerzo, se aleja”. (Entrevista huertero $N^{\circ} 4$ )

"No lo tenemos organizado, diagramado (...) se fue dando todo como espontáneo, la gente va, ve que es lo que hay que hacer, no tenemos una organización y para mi es algo que tenemos que trabajar, si bien el proyecto es colectivo no hay una organización colectiva y por eso también la huerta está como ahi (...)” (Entrevista huertero $N^{\circ} 3$ ) 
Encontramos que el disfrute en lo personal deviene del conectarse con la tierra, desconectarse de la velocidad y vorágine de la vida en la ciudad y reconectar con prácticas históricas y constitutivas de la humanidad:

Me pinta contarte todo lo otro, venir y poner la pava esperando que se junte el grupo que no es trabajo en realidad (...) si te relajas trabajando y trabajas relajado no es trabajo, si vos disfrutas son la misma cosa (Entrevista huertero $\left.N^{\circ} 1\right)$

...Creo que cada persona va aprendiendo e incorporando a su vida de acuerdo a sus experiencias y lamentablemente, en este sistema que excluye a la gente, se va perdiendo esa información. Y bueno un poco la agroecología trata de eso, de poder rescatar esos saberes, poder reincorporar al sistema esos conocimientos que acompañaron milenariamente al hombre, el hombre y la agricultura o la humanidad y la agricultura fue algo que estuvo siempre presente y que bajo este modelo quedo muy excluido, escondido. (Entrevista huertero $N^{\circ} 2$ )

Se hallan en los relatos una multiplicidad de valoraciones por parte de los huerteros, comenzando por el valor intrínseco del espacio en sí y el reflejo que encuentran allí, de una alternativa productiva, de una construcción colectiva, histórica y contextualizada del aprendizaje; lo cual asociamos a los aspectos motivacionales para llevar adelante la promoción de prácticas y saberes colectivos. Considerando aquí el hecho de potenciar las posibilidades de intercambio con otras personas incluso externas al espacio.A continuación algunos fragmentos que dan cuenta de esto:

Algo a tener en cuenta es cuidar a las personas que participan ahí y en este sentido la retribución (Entrevista huertero $N^{\circ} 3$ )

Cambiabamos verduras de la huerta con gente del instituto por productos, por alimentos, aceite yerba, arroz (Entrevista huertero $\left.N^{\circ} 1\right)$

Las capacidades que los participantes mencionan y que consideramos como extrapolables a otros contextos de sus vidas son amplias, uno de los entrevistados manifestó no saber precisar en palabras cuáles eran los aprendizajes personales que surgen de su participación en la huerta, pero siempre resaltando que su vida diaria había sido modificada por estos aprendizajes en un sentido positivo. Algunas de las capacidades mencionadas son: ingenio, solución de problemas con material de descarte, la capacidad de observación y la paciencia, en palabras de uno de ellos: "la complejidad del todo".

En todos los casos se resalta el aprender haciendo, donde no se considera al error como fracaso si no como parte del proceso: 
En el peor de los casos no te sale, y terminas aprendiendo igual. (Entrevista huertero $N^{\circ} 3$ )

Con las plantas me di cuenta que aprendes mientras te vas equivocando y me gusta ver como vienen personas nuevas y como con el tiempo van ganando confianza porque me veo hiperreflejado, dudaba no quería hacer (...) aprendes cómo funcionan las cosas haciendo, eso es una gran forma de aprender" (Entrevista huertero $N^{\circ} 1$ )

En algún momento cuando estaba más activo en la huerta me he dedicado a estudiar sobre el cultivo, ver plantas en particular y hasta ahi, despues ya ha sido aprendizaje empírico, en la práctica. (Entrevista huertero $N^{\circ} 4$ )

Estos relatos ponen de manifiesto la espontaneidad del proceso de aprendizaje autodirigido como un proceso de participación gradual, donde los participantes aprenden y se involucran mientras van adquiriendo confianza e independencia, hasta alcanzar una participación plena que sigue el ritmo del grupo.

Finalmente, concluimos que las prácticas y el consecuente aprendizaje que se desarrolla en este contexto informal, nace del disfrute, la perseverancia, el compartir y el vínculo; y no se encuentran nunca aislados de la realidad socio-política.

\subsection{Prácticas generadoras de aprendizaje:}

Se analizan en esta dimensión aquellas prácticas aprendidas en función de cómo es un día en la huerta y que aspectos de estas prácticas se constituyen como aprendizajes para toda la vida.

En este sentido, las prácticas en sí son múltiples y diversas:

Primero llego, doy una vueltita para mirar, miro las novedades y prendo la manguera, dejo regando (...) voy a buscar un lugar en el que he estado y continúo la actividad que había dejado o empiezo otra (Entrevista huertero $N^{\circ} 4$ )

Hay distintas formas, también tocó que tenía una hora al medio y me venía a hacer cosas puntuales como traer la basura organica o hechar una aguita, cosas asi o nos dejábamos trabajo preparado del uno para el otro (...) seguimos un calendario muy tranqui, el calendario biodinámico tratamos de organizar nuestras actividades en relación a ese calendario (...) hay una banda de cosas para hacer, siempre se puede hacer todo lo que la huerta demanda, trasplantar regar, cosechar, aporcar, ingresar basura que viste (...) comemos, cortamos a comer, siempre el momento de la comida es muy importante (Entrevista huertero $N^{\circ} 1$ ) 
Otras actividades mencionadas fueron alimentar a las lombrices, limpieza $\mathrm{u}$ ordenamiento de canteros con un criterio determinado, respetando interacciones entre distintos cultivos y con otras plantas que crecen espontáneamente y pueden ser benéficas para la huerta, siembra o cosecha.

Nos la pasamos sacando yuyos, la falsa idea de que controlas algo" (Entrevista huertero $\left.N^{\circ} 1\right)$

Para hacer la huerta uno tiene que ir proyectándola, haciendo los plantines que después vas a trasplantar cuando coseches lo que ya está. (Entrevista huertero $N^{\circ} 3$ )

Todas estas prácticas sustentan las capacidades aprehendidas mencionadas por los participantes como la observación o la paciencia.

Una característica de las prácticas que se desprendió de varios de los relatos fue que lo ideal era comenzar la jornada de trabajo por la mañana muy temprano y si es posible dedicarle varias horas; encontramos aquí un posible reflejo del entusiasmo que genera aprender por interés propio.

Por otro lado, los entrevistados perciben sus prácticas como "sencillas", en sus palabras:

Yo no creo que sea mucha ciencia cultivar. Si hay mucha ciencia detrás para optimizar, para conocer más, pero me parece que es algo sencillo en el sentido de que tú plantas y pueden crecer las cosas, no hay que estudiar para iniciarse (Entrevista huertero $N^{\circ} 4$ )

Denotando aquí que aunque las prácticas agroecológicas y los aprendizajes asociados a estas conlleven un esfuerzo (físico, de planificación, organización, etc), no son pensadas como trabajo forzoso por quienes las realizan, más bien las conciben como un modo de vida y a su vez entendiendo a la agroecología como una práctica política, nos adentramos en la última categoría de análisis.

\subsection{Dimensión político-social}

En esta categoría se busca identificar cual es el grado de vinculación que los participantes vislumbran entre sus prácticas y aspectos macrosociales, económicos, políticos y a su vez el nivel de percepción sobre estas dimensiones. Con esta valoración se intenta conocer el punto de vista sobre el concepto de soberanía alimentaria, teniendo en cuenta las consideraciones y expectativas sobre sus prácticas, pudiendo considerarlas como potenciales fuentes de cambio social en contextos globales.

A partir de todas las entrevistas se pudo observar la relevancia y vinculación de las prácticas llevadas a cabo en la huerta con el concepto de soberanía alimentaria y desde este perspectiva, la construcción de una soberanía en términos más holísticos y orientada a la autonomía en las formas de producción, consumo y comercialización. 
En concordancia con el planteo de MEJIA ARAUZ (2005) propuesto al inicio de esta investigación, los entrevistados sitúan su aprendizaje no sólo como un proceso participativo y colectivo a escala comunitaria reducida, sino también como aprendizajes fundados en valores que se corresponden con un marco sociocultural preexistente, el cual a su vez estimula la construcción de saberes trascendentales que tengan un impacto en ese contexto, es decir, que asocian directamente sus prácticas y aprendizajes al ejercicio político de la agroecología, entendiendo a la política como maneras de organizar la vida en sociedad y haciendo hincapié en aspectos fundamentales de esta organización como lo es la distribución de los alimentos en función de modelos productivos a gran escala. En palabras de los entrevistados:

Este aprendizaje de la experiencia productiva de una huerta así, a pulmón, muy distinta a todo lo que produce alimento en todos lados o el alimento que nos llega a la mesa, todo ese aprendizaje ¿no? ¿de donde sale el alimento que nos llega a la mesa? ¿cómo se produce? ¿que nos alimenta? (...) ¿Cuanto vale el tomate? Si yo te tengo que cobrar un tomate desde que pongo la semilla y vengo a cuidarlo (...) al menos tres meses ¿cómo hago yo para que en ese tomate se vea reflejado ese trabajo y ese tiempo en el valor de la fruta? Para mi, ¿que precio tiene? Ninguno. Crece, el alimento está ahí disponible, no debería ser una cuestión asociada al dinero, pero si esta para cuestionarse todas estas cosas (formas de producción). (...) (Entrevista huertero $N^{\circ} 1$ )

Hay una vinculación muy directa y es un trabajito cotidiano también, nunca llegamos a resolver completamente la situación porque uno entiende que soberanía alimentaria realmente existe bajo un modelo de estado, vida y comunidad en donde estén garantizados ciertos derechos. La soberanía alimentaria es un derecho y como derecho hay que conquistarlo y construirlo, hacerse cargo de esa responsabilidad. Bueno, tenemos la conciencia de que tenemos que aprender a alimentarnos sin envenenarnos y que podemos aportar cada uno de nosotres a la soberanía alimentaria, creo que este espacio ayuda muchísimo a poder pensar eso... (Entrevista huertero $N^{\circ}$ )

(este espacio tiene...) Gran valor de resistencia y siempre enlazada con otras resistencias. Es una práctica anticapitalista ese es mi fundamento para hacer agroecología y la salud también. (...) Tener tu huerta y producir tus verduras es el mejor ejemplo de lo que significa la soberanía alimentaria, por que vos vas poder elegir qué plantar, en qué momento, porque vos vas a ser el señor y soberano o soberana de tu forma de producir de tu forma de consumir, de tu forma de comercializarlo. (...) la soberanía alimentaria va de la mano con la soberanía de todo, soberanía sobre nuestras vidas, cuerpos, etc., por eso digo que no es algo aislado, no sirve de nada tener soberanía alimentaria si no somos coherentes con todas las otras. (Entrevista huertero $N^{\circ} 4$ )

Todos los relatos anteriores dan cuenta de la responsabilidad político-social que se enfrenta al construir y habitar espacios con estas características. Siendo el eje principal de estas prácticas la alimentación, una necesidad básica y constitutiva de la existencia, así como una condición innegociable para llevar a cabo una vida digna. 
Además, frente a la expectativas que los participantes tienen para con la huerta, resalta la importancia de la sostenibilidad del mismo:

No nos podemos dormir, este espacio se construye todos los días con laburo voluntario, estratégico porque tenemos que sortear toda la burocracia y trabas físicas (Entrevista huertero $N^{\circ} 1$ )

"Ya no tiene vuelta atrás este espacio, me parece que tiene un crecimiento, un aporte y una energía muy hermosa que va a hacer que esto siga girando, siga creciendo, se siga expandiendo y que está pasando en todos lados, no estamos tan aislados en el sentido de que no somos los únicos que estamos construyendo huertas en las ciudades, hay mucha gente que está en la misma en la ciudad, construyendo su huerta de manera comunitaria, autogestiva; y un poco también nuestra impronta y esperanza es que esos vínculos y articulaciones sean cada vez más fuertes para que todos los espacios de agricultura urbana se sigan sosteniendo y vayan creciendo en valor; estamos en un momento en el contexto del país con emergencia alimentaria, la gente no accede a la los alimentos básicos, a los nutrientes básicos para llevar una vida digna y es una responsabilidad muy grande por parte del estado poder garantizar una alimentación sana y concisa y en este momento lo estamos haciendo huerteres en nuestros territorios, en nuestros baldios, en nuestras vias, en nuestros descampados. (Entrevista huertero $\mathrm{N}^{\circ} 2$ )

\section{Discusiones}

En relación a los aspectos predominantes del aprendizaje en un contexto informal como la huerta en la cual se realizó la investigación, se puede hacer las siguientes caracterizaciones y reflexiones.

La forma de aprendizaje que se genera en la huerta, es claramente de carácter participativo, gradual y empírico. Las prácticas asociadas a los aprendizajes están fundadas en el interés propio de cada uno de los participantes, en el asombro y la espontaneidad que nutre la autodirección de los procesos de aprendizaje, a su vez ligadas al disfrute del vínculo con la tierra y con otros. Toma fundamental relevancia el aspecto colaborativo ya que los aprendizajes que aquí tienen lugar están fuertemente vinculados a la afectividad, a los valores y a la cooperación entre pares.

Dentro de este panorama, se propone un manejo ecológico de los recursos naturales mediante formas de acción social colectiva para el desarrollo de sistemas de control participativo y democrático, en los ámbitos de la producción y circulación, que impliquen una visión agroecológica integradora como una praxis (LLERENA DEL CASTILLO; ESPINET BLANCH, 2017).

De la mano de esto vemos grandes aprendizajes que pueden ser los pilares para construir un modo de habitar el mundo de manera sustentable y equitativa, en función de las manifestaciones que aquí encontramos de apertura a la empatía, percepción sensible de 
los vínculos con otros seres, perseverancia, desarrollo de la capacidad de observación y la paciencia, como también la coexistencia tendiente a la cooperación. Todo esto responde a la propuesta de DELORS (1996): Aprender a conocer, aprender a hacer, aprender a vivir juntos y aprender a ser, y se asocia al planteo de ACEVEDO-OSORIO (2013) de que la educación agroecológica es vista como el principal instrumento de transformación.

La gran implicancia política de los aprendizajes en este contexto informal ayuda a pensarnos como soberanos de nuestros sistemas de producción y de consumo. La falta de calidad en la producción alimenticia y su mala distribución debido al modelo productivo que hoy rige en Argentina y el mundo, constituyen la base de las injusticias estructurales de una sociedad en donde el acceso al alimento de calidad es un privilegio. Los saberes que se entretejen en esta huerta apelan a la conciencia y a la construcción de derechos en pos de lo que muchas comunidades denominan "el buen vivir", lo que se sostiene necesariamente con responsabilidad social y conciencia de clase. En este sentido, SÁNCHEZ DE PRAGER ET AL. (2015) en un estudio de familia campesinas enfatizan precisamente en los contextos de capacitación no formales e informales, y añadiendo sobre la necesidad e importancia de la autonomía y seguridad alimentaria en la implementación de sistemas productivos alternativos.

La agroecología como ejercicio político es una herramienta para sentipensar, en palabras de ESCOBAR (2016), una nueva dirección para la estructura epistémica moderna, la cual se ha basado históricamente en el borramiento efectivo de los saberes populares, el pensamiento crítico y la participación activa de las personas en sus propios territorios. Estos procesos han tenido un impacto catastrófico y desesperanzador hasta la actualidad en relación a las posibilidades de transformación social. Es por ello que es indispensable poner una pausa a estas lógicas y centrar la atención en la revalorización de los espacios alternativos en los cuales se tejen saberes y modos de horizontales de estructuración y construcción social que nos desafían a tomar parte en nuestro mundo y hacerlo un lugar más habitable.

Como desafío colectivo, la idea es seguir aprendiendo de estos espacios, y para ello concluimos con algunas de las preguntas de ESCOBAR (2016) para "entender a la naturaleza" de la cual somos parte: ¿Como re-aprendemos a inter-existir con todos los humanos y no-humanos? ¿Debemos recuperar cierta intimidad con la Tierra para reaprender el arte de sentipensar con ella? ¿Cómo hacerlo en contextos urbanos y descomunalizados? 


\section{Referencias Bibliográficas}

ACEVEDO-OSORIO, A. Escuelas de agroecología en Colombia. La contrucción del conocimiento agroecológico en manos campesinas. En: ALTIERI, M.; SARANDON, S; MORALES, C.; FUNES, F.; SUIRA, S. (Ed.) CONGRESO LATINOAMERICANO DE AGROECOLOGÍA, 4., Lima, Peru, 2013. Anales... Lima, Peru: Sociedad Científica Latinoamericana de Agroecología (SOCLA), 2013. Disponível em: https://orgprints.org/view/projects/int-conf-socla-2013.html

DELORS, J. La educación encierra un tesoro. Madrid: Santillana. 1996.

DUSSI, M. C., FLORES, L. B.; BARRIONUEVO, M. E. Agroecología y educación: multidimensión en la comprensión de sistemas complejos en Patagonia. En: JORNADAS NACIONALES DE EXTENSIÓN RURAL Y IX DEL MERCOSUR: EL ENCUENTRO EN LA DIVERSIDAD, 17., Santa Fé, Argentina, 2014. Anales... Santa Fé. Argentina: Zavalla, 2014. p. 1015-1019.

ESCOBAR, A. (2016). Desde abajo, por la izquierda y con la tierra. https://elpais.com/elpais/2016/01/17/contrapuntos/1453037037_145303.html Acceso en: 30 de junio 2020.

GUBER, R. La etnografia, metodo, campo y reflexividad. Buenos Aires: Siglo Veintiuno, 2011.

LLERENA DEL CASTILLO, G.; ESPINET BLANCH, M. Agroecología escolar. Espana: Pollen, 2017.

MARTIN, R. B. Perspectiva teórica sobre el estudio de los contextos y comunidades de aprendizaje. En: MARTÍN, R, RINAUDO, M.C. y PAOLONI, P. V. Comunidades.

Estudios y experiencias sobre contextos y comunidades de aprendizaje. Argentina: Eduvim, 2019, p. 75-13.

MARTÍN, R. B. 2014. CONTEXTOS DE APRENDIZAJE. Formales, no formales e informales. IKASTORRATZA e-Revista de Didáctica, v.12, 2014. Disponible en: http://www.ehu.eus/ikastorratza/12_alea/contextos.pdf Acceso en: 30 de junio 2020.

MARTÍN, R.B.; PALOMBO, N.; PERRONI GASULL, C.; PAREDES MALDONADO, Y.; SILVERA RUIZ, L.; SAGO HERRADOR, E. y CHIAPERO, F. La investigación educativa en diversos contextos de aprendizaje. Una experiencia de formación docente. En JORNADA DE EXPERIENCIAS E INVESTIGACIONES EDUCATIVAS EN CIENCIAS EXACTAS Y NATURALES, 1., 2019. Anales...Córdoba, Argentina. 2019.

MEJIA-ARAUZ, R. Tendencias actuales en la investigación del aprendizaje informal. Sinéctica, v.26, 2005. Disponible en: https://sinectica.iteso.mx/index.php/SINECTICA/article/view/246/239 Acceso en: 30 de junio 2020 
ROGOFF, B. The cultural nature of human development. Nueva York: Oxford Univ., 2003.

SANCHEZ DE PRAGER, M.; CASTELLANOS SIERRA, M.; CÁRDENAS HENAO, J.; ZAPATA, W.; CASTILLO ORDOÑEZ, E.; VELEZ ZABALA, F. ; GÓMEZ DUQUE, S. Aprendizajes en experiencia de apropiación de conocimiento (APS) agroecológico en familias campesinas del Valle del Cauca (Colombia). CONGRESO LATINOAMERICANO DE AGROECOLOGÍA - SOCLA, 5., La Plata, 2015. Anales... La Plata, 2015. Disponible en: http://sedici.unlp.edu.ar/handle/10915/54447 Acceso en: 30 de junio 2020.

TRILLA, J.; GROS, B.; LÓPEZ, F. y MARTÍN, M. J. La educación fuera de la escuela. Ámbitos no formales y Educación Social. Barcelona, España: Ariel Educación. 2003.

VIA CAMPESINA. Movimiento Campesino Internacional. Disponible:

https://viacampesina.org/es/quignifica-soberanalimentaria/ Acceso en: 23 de junio 2020. 